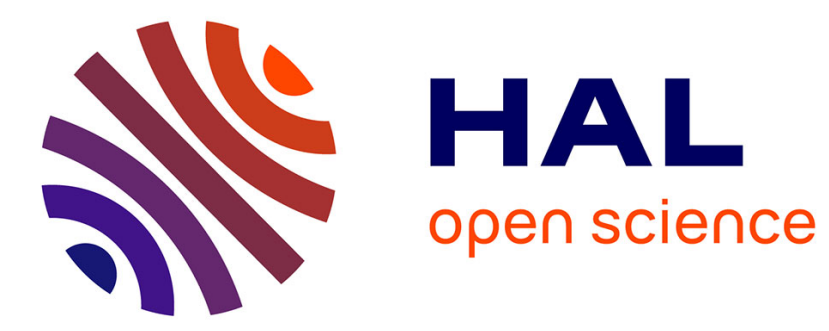

\title{
L'apparition des attributs individuels des saints dans l'art médiéval
}

\author{
Charlotte Denoel
}

\section{To cite this version:}

Charlotte Denoel. L'apparition des attributs individuels des saints dans l'art médiéval. Cahiers de Civilisation Médiévale, 2007, 50 (198), pp.149-160. hal-00865748

\section{HAL Id: hal-00865748 https://hal-bnf.archives-ouvertes.fr/hal-00865748}

Submitted on 25 Sep 2013

HAL is a multi-disciplinary open access archive for the deposit and dissemination of scientific research documents, whether they are published or not. The documents may come from teaching and research institutions in France or abroad, or from public or private research centers.
L'archive ouverte pluridisciplinaire HAL, est destinée au dépôt et à la diffusion de documents scientifiques de niveau recherche, publiés ou non, émanant des établissements d'enseignement et de recherche français ou étrangers, des laboratoires publics ou privés. 


\section{L'apparition des attributs individuels des saints dans l'art médiéval}

A l'époque médiévale, les attributs, qui faisaient partie du langage des signes auquel les artistes avaient recours pour exprimer une idée, une situation ou raconter une histoire, revêtaient une grande importance en Occident dans les représentations des saints, dont ils indiquaient l'identité aux fidèles. Leur diffusion progressive dans les images est allée de pair avec l'inflation du culte des saints durant les derniers siècles du Moyen Age. Jusqu’à présent, les études consacrées à l'iconographie des saints se sont attachées, les unes à décrire ce processus de diffusion dans l'art, les autres à recenser sous la forme de dictionnaires les attributs des saints et à identifier précisément leur signification ${ }^{1}$. Il est cependant un autre aspect de la question qui demeure encore largement inexploré, celui de la date et des origines de l'apparition des attributs individuels des saints dans l'art occidental. Pourtant, faire la lumière sur ces deux points permettrait de mieux saisir la signification et le rôle des attributs dans l'art et dans la société comme de mieux cerner la nature des relations qui unissaient les fidèles aux œuvres d'art. Tel sera l'objet de notre propos dans les lignes qui suivent : une fois retracée la chronologie de l'apparition des premiers attributs individuels, nous nous efforcerons de replacer ce phénomène dans son contexte religieux et social, en insistant sur la réflexion théologique qui le sous-tend et sur les changements sociaux survenus à l'époque où les attributs commencèrent à se répandre dans l’art.

Les attributs qui servent à caractériser les saints peuvent être divisés en trois grandes catégories, définies par Louis Réau dans sa monumentale Iconographie de l'art chrétien ${ }^{2}$ : les attributs universels, génériques et individuels. Ces derniers, qui forment de loin la catégorie la plus diversifiée, se rapportent à un épisode de la légende ou du martyre des saints, à un aspect de leur culte, voire encore à leur nom. Leur stock, qui n’a cessé de s'enrichir au cours des derniers siècles du Moyen Age, a suivi l'évolution du culte des saints, de nouveaux attributs venant s'ajouter aux précédents à l'occasion de la canonisation de nouveaux saints et de l'éclosion de nouveaux cultes en Occident. Contrairement aux attributs universels et génériques, cette dernière catégorie

\footnotetext{
${ }^{1}$ Concernant l'historiographie des travaux sur les attributs des saints, nous nous permettons de renvoyer le lecteur à notre article sur «La naissance de l'iconographie religieuse au XIXe siècle. Le milieu des archéologues et des sociétés savantes » (Bulletin du Comité des Travaux historiques et scientifiques, t. 31, 2004).
} 
d'attributs est apparue tardivement dans l'art d'Occident, à l'exception d'un petit noyau d'attributs, parmi lesquels figurent les clefs de saint Pierre, les symboles des évangélistes, l'agneau de sainte Agnès et le dragon de saint Michel. Ceux-ci reçurent dans la Bible une interprétation symbolique; c'est pourquoi ils furent introduits dans l'art à une date ancienne, dès l'époque paléochrétienne, et traversèrent les siècles, sans aucune modification du stock initial. Si ces motifs peuvent être considérés comme des signes personnels, la dimension symbolique qu'ils revêtaient dans les images l'emporta néanmoins sur leur caractère identitaire jusqu'au Moyen Age central. Un changement se produisit durant les dernières décennies du XIIe siècle, lorsque les artistes commencèrent à associer aux saints des attributs individuels en vue de les identifier avec certitude. On assiste ainsi à cette époque au passage d'un système iconographique abstrait, fondé sur la valorisation du statut spirituel du saint, à un nouveau système iconographique plus concret, au sein duquel l'accent est mis sur les aspects biographiques et terrestres de la vie du saint. Dotés de caractéristiques individuelles destinées à mettre à la portée d'un grand nombre les vertus héroïques et les hauts faits accomplis par les serviteurs du Christ, ces derniers revêtent ainsi aux yeux des fidèles une allure plus familière.

Ces allusions nouvelles à la condition terrestre des saints dans les images, si elles demeurent encore discrètes durant la seconde moitié du XIIe siècle, ne cessent par la suite de se multiplier, les attributs individuels envahissant les représentations des saints au cours du XIIIe siècle et aux siècles suivants. Une enquête menée à partir d'un échantillon de 320 manuscrits enluminés contenant des représentations de saints, produits entre l'époque carolingienne et le XVIe siècle et issus de différentes aires géographiques (France, Angleterre, Pays-Bas, Belgique, Allemagne, Italie, Espagne), a permis de mettre en évidence ce phénomène, qui va de pair avec le développement du culte des saints. Dans les manuscrits, celui-ci se traduit notamment par une plus grande place réservée aux images des saints. Peu développée entre l'époque carolingienne et la fin du XIIe siècle, l'illustration hagiographique concernait principalement les passionaires et les vies de saints locaux et se limitait aux représentations des miracles et des supplices. Dès la fin du XIIe siècle, en revanche, l'apparition de nouveaux livres de prière, tels que le psautier-heures à l'usage des laïcs, et, au siècle suivant, de légendiers en latin et en langue romane, a entrainé le développement des représentations individuelles de saints, désormais pourvus d’un ou plusieurs attributs destinés à faciliter leur identification.

L'apparition des attributs individuels, intervenue durant la seconde moitié du XIIe siècle, s'est faite progressivement, et non brutalement, comme le montrent les rares exemples fournis

${ }^{2}$ Louis Réau, Iconographie de l'art chrétien, I, Paris, 1955, p. 416-430. 
par la sculpture de l'Ouest de la France ${ }^{3}$ : les églises de Pont-l'Abbé d'Arnoult et de Corme-Royal, en Charente-Inférieure, qui sont toutes deux des dépendances de l'abbaye Sainte-Marie-auxDames de Saintes, bâties, la première entre 1140 et 1170, la seconde à une date indéterminée durant la seconde moitié du XIIe siècle, sont ornées de statues de saints pourvus d'attributs les différenciant des autres personnages sculptés. Dans une archivolte du tympan central du portail Ouest de Pont-l'Abbé, on distingue en effet sainte Catherine, saint Laurent et saint Paul, identifiables à leurs attributs respectifs, une roue, un gril et une épée. A Corme-Royal, on reconnait également la figure de sainte Catherine grâce à la roue de son supplice qu'elle exhibe devant elle aux côtés de saints guerriers, dans l'archivolte d'une arcade de la façade Ouest ${ }^{4}$.

Néanmoins, ces exemples demeurent encore isolés dans la seconde moitié du XIIe siècle, comme en témoigne l'iconographie d'un livre d'images probablement originaire de Saint-Bertin, vers 1200 (La Haye, KB, 76F5), qui traduit bien les hésitations des artistes vis-à-vis du nouveau procédé iconographique au commencement du XIIIe siècle : si saint Denis (f. 28v) et sainte Marguerite (f. 32, ill. 1) sont figurés avec leurs attributs respectifs, la tête entre les mains et le dragon, d'autres saints tels que Gilles (f. 33v, ill. 2), Marie-Madeleine (f. 34v), Maurice d'Agaune (f. 35v), Ambroise (f. 38v) ou Augustin (f. 38v), sont encore pourvus d'attributs génériques qui ne permettent guère de les identifier. Les décennies suivantes voient cependant ces attributs se multiplier et se répandre dans toutes les formes d'art. Dans les manuscrits, les attributs se sont largement diffusés dans les vies de saints et dans les psautiers, dont la production connut un essor sans précédent au XIIIe siècle - en particulier dans les centres de Paris, Metz, Liège, Bruges et Gand -, pour satisfaire une demande croissante de la part des élites laïques, nobles et bourgeois. Le contenu et les illustrations du psautier, auparavant réservé à un usage strictement liturgique, furent ainsi adaptés aux pratiques de piété personnelle de ces nouveaux lecteurs, avec notamment l'apparition, dès le tournant du XIIe siècle, de représentations de saints avec leurs attributs ou de scènes de leur martyre ${ }^{5}$. Une image de saint Denis portant sa tête entre les mains dans le psautier de Huntingfield, probablement exécuté à Oxford vers 1210-1220 (New York, Pierpont Morgan Library, M 43, f. 24v), constitue l'un des plus anciens témoins de ce type d'illustration. D'après Elisabeth Carlvant, les cycles d'apôtres, apparus dès la seconde moitié du XIIe siècle en Flandre, puis dans les psautiers exécutés aux alentours de 1250 par les enlumineurs du groupe tournaisien

\footnotetext{
${ }^{3}$ A cet égard, le CD-Rom de l'Index of Christian Art, dont la Bibliothèque Byzantine à Paris conserve un exemplaire, nous a été très utile. Nos recherches iconographiques ont été conduites à partir d'un corpus restreint de saints et saintes, que nous nous sommes efforcée de rendre homogène.

${ }^{4}$ Cf. Arthur K. Porter, Romanesque Sculpture of Pilgrimage Roads, Boston, 1923, t. 7, pl. 1004 et 1013.

5 Le renouvellement de l'illustration des psautiers au XIIIe siècle s'inscrit dans l'essor général de la narration hagiographique à cette époque. On trouve ainsi de nombreuses scènes narratives illustrant les vies de saints dans les livres d'images, notamment dans les manuscrits suivants : Amiens, BM, ms. 108 (Bible de Sancho le Fort, Navarre,
} 
d'Eerste, sont à l'origine de ces images hagiographiques narratives ${ }^{6}$. Alors que, dans la plupart de ces cycles, les apôtres sont encore indifférenciés, un changement notable se produit dès les années 1260, avec les œuvres du groupe de Tweede, actif à Gand et à Bruges entre 1260 et 1290/95, qui contiennent plusieurs initiales historiées figurant sainte Marguerite émergeant triomphalement des entrailles du dragon qui l'a dévorée ${ }^{7}$. Bientôt adoptés par les enlumineurs des régions voisines ${ }^{8}$, ces cycles hagiographiques ont certainement contribué à populariser les attributs des saints dans l'art. Dans le domaine de la sculpture, on trouve également des figures de saints avec leurs attributs aux portails des cathédrales gothiques, notamment à Notre-Dame de Chartres, où, vers 1200, les ébrasements du portail du Couronnement, au Nord, et du portail central méridional accueillent respectivement les statues des précurseurs et celles des apôtres (ill. 3), pour la plupart reconnaissables à leurs attributs individuels. Ajouté quelques années plus tard, le portail des Martyrs, au Sud, contient, aux ébrasements, les représentations des saints Denis, Vincent de Saragosse et Piat de Seclin, se tenant debout sur leurs attributs distinctifs, le lion pour Denis, le corbeau et le loup pour Vincent, et le gouverneur Rictiovarius portant une couronne pour Piat (ill. 4). Non loin de là, on retrouve saint Vincent, avec cette fois avec une crosse et le sommet de sa tête dans les mains. La peinture murale a également accueilli ces nouveaux motifs iconographiques, ainsi qu'en témoigne l'exemple de saint Christophe, qu'une fresque exécutée vers 1200 sur le mur extérieur Nord de l'église de Castel Appiano, en Italie, représente portant le Christ sur son bras gauche, un bâton feuillu dans la main droite.

1197), La Haye, KB, 76F5 (Livre d'images, Saint-Bertin?, vers 1200) et Paris, BnF, NAF 16251 (Livre d'images de Madame Marie, Hainaut, 1285-1290).

${ }^{6}$ Elisabeth Carlvant, Thirteenth century illumination in Bruges and Ghent, Columbia University, 1978, p. 280-285. Le prototype des cycles d'apôtres se trouve, d'après l'auteur, dans un psautier à l'usage de Gand, de la seconde moitié du XIIe siècle (Tournai, Bibliothèque capitulaire, ms. A 15), qui contient un cycle d'apôtres peints suivant l'ordre du Credo. Les personnages sont identifiables par l'inscription de leur nom.

${ }^{7}$ Sur le groupe de Tweede, voir la thèse d'Elisabeth Carlvant, Op. cit., passim. Les psautiers suivants, issus du groupe de Tweede, contiennent des images de sainte Marguerite sortant des entrailles du dragon : Londres, British Library, Burney 345, f. 86v (Gand, 1265-1275), Dublin, Chester Beatty Library, W 61, f. 95 (Bruges, 1265-1275), Oxford, Bodleian Library, Auct. D. 4. 2., f. 87v (Bruges, 1260-1270). Ainsi que l'a souligné Elisabeth Carlvant (p. 339-341), Marguerite d'Antioche est la seule sainte à être identifiable dans les initiales historiées illustrant ce groupe de psautiers. Il se peut ainsi que des femmes aient été les destinataires de ces psautiers, car sainte Marguerite était fréquemment invoquée par les femmes enceintes, l'épisode de la sainte émergeant triomphalement du dragon évoquant les douleurs de l'accouchement.

${ }^{8}$ On rencontre des cycles de saints similaires dans les psautiers du groupe de Dampierre, produits dans le diocèse de Thérouanne durant les trois dernières décennies du XIIIe siècle, et dont le psautier de Guy de Dampierre, vers 1260 1275, est le principal représentant (Bruxelles, Bibliothèque Royale, ms. 10607), ainsi que dans les psautiers exécutés en France du Nord (cf. Paris, Bibliothèque Sainte-Geneviève, ms. 2689 : Psautier de Genlis, vers 1260) et dans la région de Liège durant la seconde moitié du XIIIe siècle (cf. Paris, BnF latin 1077, vers 1265-1275; Cambridge, Fitzwilliam Museum, ms. 288 : Psautier de Lambert le Bèque, vers 1280-1290 ; New York, Pierpont Morgan Library, M 183 : Psautier-Heures de Lambert le Bèque, vers 1280-1290; Liège, Bibliothèque de l’Université, ms. 431, vers 1280-1290). Dans la plupart des exemples illustrant les deux derniers groupes de manuscrits, les saints sont individualisés à l'aide de signes distinctifs, tels les oiseaux rassemblés derrière saint François dans cette initiale anonçant le psaume 2 d'un Psautier-Heures à l'usage de Liège (Paris, BnF latin 1077, f. 16). 
Empruntés à différentes formes d'art, ces exemples indiquent clairement que les attributs sont devenus un élément constitutif de l'iconographie des saints dans le courant du XIIIe siècle. A cet égard, il est intéressant de constater que ce phénomène concerne avant tout le Nord de l'Europe, d'où sont issues la plupart des œuvres mentionnées, à quelques exceptions près.

Selon l'explication généralement avancée pour expliquer l'origine de l'apparition de ces motifs dans l'art, la statuaire gothique aurait été la première, au commencement du XIIIe siècle, à figurer les attributs individuels des saints pour pouvoir identifier ceux-ci, à défaut de l'inscription du nom des saints, ceux-ci s'avérant difficilement lisibles pour les fidèles à l'échelle de la sculpture monumentale 9 . Habituellement employé dans les autres formes d'art, ce procédé permettait aux artistes d'identifier les saints en l'absence de signe particulier. Toutefois, cette thèse mérite d'être nuancée, car, non seulement l'apparition des attributs individuels a précédé l'éclosion de la statuaire gothique, mais encore les artistes n'avaient pas systématiquement recours à l'inscription des noms pour préciser l'identité des saints avant le XIIIe siècle, ceux-ci restant souvent indifférenciés aux yeux des fidèles. Le contexte de l'époque ne pourrait-il plutôt contribuer à expliquer les changements survenus dans l'iconographie des saints à partir de la seconde moitié du XIIe siècle ? Ceux-ci se situeraient alors au point de rencontre de différents phénomènes religieux et sociaux.

L'accent mis sur la condition terrestre du saint à travers la figuration d'attributs individuels est intervenue à une époque où la nécessité d'évangéliser davantage de fidèles se fit de plus en plus pressante, devant la propagation des hérésies. En effet, les années qui suivent la tenue du troisième Concile de Latran (1179) virent s'opérer de nombreux changements au sein de l’Église: destiné à relancer l'idée de croisade et à combattre les nombreuses hérésies qui représentaient un danger permanent pour l’Église catholique (Albigeois, bandes armées, Vaudois...), ce concile constitue une étape essentielle entre la Réforme grégorienne du XIe siècle et la Réforme pastorale du XIIIe siècle ${ }^{10}$. La pratique l'emportant sur la théorie et sur les

\footnotetext{
${ }^{9}$ Cf. notamment pour cette interprétation, les travaux d'Émile Mâle, qui met en relation l'apparition de la statuaire gothique et le développement des caractères et des emblèmes des saints (L'art religieux du XIIIe siècle en France, Paris, 1905, p. 322-353), et de Guillaume de Jerphanion, qui estime que les attributs ont été inventés en Occident avec la sculpture gothique, car celle-ci ne pouvait recevoir d'inscription désignant les personnages représentés («Les caractéristiques et les attributs des saints dans la peinture cappadocienne », dans Analecta Bollandiana, LV (1937), p. 128).

${ }^{10}$ Cf. sur ce concile, Le troisième concile de Latran (1179), dir. Jean Longère, Paris, 1982, et en particulier la contribution de Joseph Avril, «L'encadrement diocésain et l'organisation paroissiale », p. 53-74.
} 
controverses théologiques qui animaient l'époque, priorité fut donnée au cours du concile aux prescriptions d'ordre disciplinaire et pastoral. L'accent fut notamment mis sur la morale des clercs, les visites pastorales et la gratuité de l'enseignement et de la licentia docendi. Les décisions du concile furent par la suite reprises dans les statuts synodaux de la fin du XIIe siècle, puis complétées lors du quatrième concile du Latran ${ }^{11}$, le ministère pastoral constituant l'un des volets essentiels de la législation canonique adoptée en 1215 : insistant sur l'importance de l'action pastorale auprès des fidèles, le canon 10 décrète l'institution de prédicateurs diocésains dans les églises cathédrales et collégiales. Ces auxiliaires des évêques devront les assister dans le ministère de la prédication, «visiter à leur place les populations à eux confiées lorsqu'ils ne pourront le faire eux-mêmes, les édifier par leur parole et leur exemple, leur dispenser, s’ils en ont besoin, les secours utiles, de sorte que la privation des biens nécessaires n'oblige pas à l'abandon de l'œuvre entreprise. $»^{12}$ Pour que les clercs puissent mener à bien leur tâche pastorale, il est nécessaire qu'ils possèdent une certaine culture, c'est pourquoi le décret suivant réaffirme la nécessité d'entretenir dans chaque église cathédrale et collégiale un maître chargé d'enseigner gratuitement la grammaire et d'autres disciplines aux clercs et aux écoliers pauvres, et en chaque métropole un maitre en théologie chargé d'enseigner l'Écriture sainte aux clercs et de les former au ministère pastoral ${ }^{13}$. C'est ainsi qu'au XIIIe siècle, l'activité pastorale devient une priorité essentielle dans la lutte contre l'hérésie et l'évangélisation des fidèles. La prédication, qui vise à délivrer aux populations un enseignement doctrinal élémentaire, joue à cet égard un rôle majeur, en particulier grâce à l'action des ordres mendiants, qui ont grandement contribué au développement du culte des saints parmi les laïcs dès le commencement du XIIIe siècle.

Tandis que le nombre de sermons rédigés à cette époque ne cesse de croitre, le genre se renouvelle, avec notamment l'établissement d'une distinction entre les sermons destinés au temps liturgique, "de tempore», et les sermons destinés à commémorer les fêtes des saints, «de sanctis $»^{14}$. Désormais, les collections de sermons prononcés en l’honneur des saints prennent de l'ampleur, ainsi qu'en témoigne la constitution par Pierre de Reims de deux recueils indépendants de sermons sur les saints vers 1230. Les fidèles sont ainsi conviés à méditer sur les exploits et les souffrances des serviteurs du Christ et sur la valeur théologique exemplaire de leur martyre. L'exemple du sermon composé par Ranulphe de la Houblonnière (v. 1225-1288) pour la fête de saint André, le 30 novembre, illustre bien ce souci de mettre l'accent sur la passion des saints ${ }^{15}$ :

\footnotetext{
11 Sur le $4^{\mathrm{e}}$ concile de Latran, voir l'ouvrage de Raymonde Foreville, Latran I, II, III et Latran IV. Histoire des cociles cecuméniques, t. 6, Paris, 1965.

${ }^{12}$ Ed. R. de Foreville, Op. cit., p. 352.

${ }^{13}$ Cf. canon 11, éd. R. de Foreville, Op. cit., p. 353.

${ }^{14}$ Cf. Nicole Bériou, «Les sermons latins après 1200 », dans B.M. Kienzle, The Sermon, Turnhout, 2000, p. 388.

${ }_{15}$ Nicole Bériou, La prédication de Ranulphe de la Houblonnière. Sermons aux clercs et aux simples gens à Paris au XIIIe s., Paris, 1987, I, p. 15-34.
} 
partant d'une citation empruntée à l'Épître aux Galates, 2, 19, «Christo sum confixus cruci », le prédicateur s'appuie sur le fait que saint André mourut sur la croix comme le Christ pour mettre en avant le mérite, l'audace et la persévérance dont il fit preuve dans ces circonstances, et inciter l'assemblée à suivre son exemple en portant la croix du Christ, qui est à la fois armure, enseignement et vie. Perceptible dans les sermons du XIIIe siècle, ce passage d'une théologie orientée vers une finalité contemplative vers une théologie plus pratique et plus concrète, qu'Auguste Châtillon a bien mis en évidence ${ }^{16}$, témoigne ainsi d'un souci pastoral plus marqué.

Dans ce contexte, l'iconographie s'avère, elle aussi, un moyen privilégié et efficace de lutter contre les errements doctrinaux et de propager une foi orthodoxe, conforme aux dogmes de l'Église catholique, les fidèles illettrés pouvant prendre connaissance du message religieux par l'intermédiaire des images qui ornaient les églises. Cette conception des images rejoint celle qu'exprima Grégoire le Grand en 600 dans sa fameuse lettre à l'évêque iconoclaste de Marseille, Serenus ${ }^{17}$. Selon Grégoire, les images revêtent une double dimension pédagogique et affective. Elles ont pour fonction, non seulement d'instruire les illettrés et de fixer dans la mémoire des fidèles les hauts faits de l'histoire sainte, qui sont actualisés par l'image, mais aussi de susciter chez les fidèles un sentiment de componction à la vue des souffrances endurées par le Christ et ses serviteurs. Or, à une époque où l'activité pastorale prenait de plus en plus d'ampleur et devenait l'une des tâches majeures des desservants, de telles conceptions ne pouvaient qu'être réaffirmées et les images acquérir un nouveau statut au sein de l’Église. Le double combat mené par l’Église contre les hérésies, d'une part, et pour l'instruction des fidèles, de l'autre, passe désormais non plus seulement par les textes officiels et les sermons, mais aussi par les images elles-mêmes, qui véhiculent les doctrines de la foi catholique. Il convient ainsi d'adapter aux fidèles le message que souhaite délivrer l'Église, de le mettre à leur portée. Cette nouvelle importance accordée aux images apparait à la lecture des théologiens de l'époque : ainsi que l'ont souligné Arthur M. Landgraf puis Beryl Smalley ${ }^{18}$, Pierre le Mangeur (1100-1178) porte un grand intérêt à l'explication littérale de l'Écriture, dans le sillage d'André de Saint-Victor, en vue d'éclairer d'un regard neuf le texte biblique et de le rendre plus accessible aux fidèles. Dans son Historia scholastica, composée vers 1170, il s'appuie sur des exemples concrets empruntés à la liturgie, aux reliques, à l'archéologie et à la topographie de la Palestine, ainsi qu'à l'iconographie, pour justifier

\footnotetext{
16 Auguste Châtillon, «Le mouvement théologique dans la France de Philippe Auguste », dans La France de Pbilippe Auguste. Le temps des mutations. Colloque international du C.N.R.S., 1980, Paris, 1982, p. 881-902.

${ }_{17}$ Epistola XIII, éd. dans PL 77, col.1128-1130. Sur la lettre de Grégoire, voir Jean-Claude Schmitt, «L'Occident, Nicée II et les images, VIIIe-XIIIe s. », dans Nicée II, 787-1987. Donze siècles d'images religieuses. Actes du colloque international Nicée II tenu au Collège de France, Paris, 2-3-4 octobre 1986, éd. F. Boespflug et N. Lossky, Paris, 1987, p. 271-302.
} 
la Parole des Évangiles et de l'Écriture sainte. Par conséquent, les images, qui transmettent un enseignement religieux, peuvent être citées comme une autorité, pour illustrer des sermons ou une discussion théologique, et nourrir la réflexion théologique elle-même. Cette nouvelle valeur accordée aux images, qui s'est accompagnée d'une réflexion théologique sur les choix iconographiques des artistes, eut une influence certaine sur les évolutions iconographiques ultérieures.

Les images faisant partie intégrante de l'œuvre pastorale entreprise par l'Église, celle-ci chercha dès la fin du XIIe siècle à exercer un certain contrôle sur l'iconographie, afin de veiller à l'orthodoxie des représentations qui ornaient les églises. L'exemple des représentations des saints est, à cet égard, instructif : c'est en effet à partir de la seconde moitié du XIIe siècle que les allusions à l'iconographie des saints deviennent plus nombreuses dans les textes liturgiques et canoniques, alors qu'elles étaient extrêmement rares durant la période précédente ${ }^{19}$. Déjà, dans la première moitié du XIIe siècle, un commentateur de l'école d'Abélard, commentant l'épître de saint Paul aux Hébreux, 10, 9, insistait sur la nécessité de peindre dans les églises saint Pierre avec ses clefs, ainsi que d'autres saints, à la place des idoles des églises païennes, pour mieux rappeler aux fidèles les hauts faits de l'histoire sainte et extirper l'hérésie : "Ita et in primitiva Ecclesia quadam dispositione factum est, que nobis, qui gentiles eramus, loco imaginum Iovis, Iunonis, quas primitus faciebamus, sanctorum imagines concessit diversas facere, qui hodie Petrum apostolorum clavigerum pingimus claves in manu tenentem et ceteros quoque sanctos iuxta eorum dignitatem. Et hec quidem causa fuit, ne videlicet ad ritum gentilitatis, ubi nati et ideo redire proni, in his detenti facile rediremus... $»^{20}$. Plus que l'allusion à l'attribut de saint Pierre, qui est apparu à une date ancienne dans l'art, l'emploi de l'expression « juxta eorum dignitatem » dans ce contexte est très intéressant. Si le terme « dignitas », qui désigne ici le symbole, l’instrument de gloire des saints, autrement dit leurs attributs, ne peut être interprété avec certitude comme une allusion à des accessoires individuels, il n’en constitue pas moins un témoignage exceptionnel sur la valeur théologique des attributs, perçus par l'auteur comme des signes manifestant le mérite et le pouvoir spirituel de leurs possesseurs. Quelques années plus tard, le théologien Jean Beleth introduit des considérations d'ordre iconographique sur les saints à l'occasion de leur fête dans sa Summa de ecclesiasticis officiis, probablement composée vers le milieu du XIIe siècle. Ainsi tient-il les

\footnotetext{
18 Arthur M. Landgraf, Dogmengeschichte der Frühscholastik, II. Die Lebre von Christus, 1, Regensburg, 1953, p. 24-26 et Beryl Smalley, "Petrus Comestor on the Gospels and his sources », dans Recherches de théologie ancienne et médiévale, 46 (1979), 84-129.

${ }^{19}$ Parmi les rares textes antérieurs connus, citons un fragment anonyme de la fin du VIIIe s. ou du début du IXe s., intitulé «De tonsura apostolorum », et conservé dans le ms. Paris, BnF latin 11561 (f. 201v). Se réclamant d'une tradition romaine, son auteur décrit brièvement les caractéristiques physiques des apôtres dans les images, mais sans faire allusion à d'éventuels attributs. Cf. sur ce fragment, André Wilmart, «Effigies des apôtres vers le début du Moyen Age », dans Revue bénédictine, 42 (1930), p. 76.
} 
propos suivants à l'endroit de saint André : «De festo sancti Andree sciendum est, quod ipse fuit niger colore, barba prolixa, statura mediocris. Hec ideo dicimus, ut sciatur, qualis in ecclesia debet depingi, quod et de unoquoque apostolorum et multorum aliorum sanctorum est sciendum similiter. Aliter enim mentiremur nos in littera laicorum silicet in picturis. $»^{21} \mathrm{Si}$ aucune allusion n'est faite aux attributs de saint André, cette citation n'en révèle pas moins le souci des théologiens de l'époque de proposer aux fidèles des images conformes à la doctrine. Ce souci devient de plus en plus manifeste vers la fin du siècle, ainsi qu'en témoigne la Summa decretorum de Huguccio de Pise, rédigée à Bologne vers 1188-1190. Ce commentateur de Gratien, qui fut évêque de Ferrare de 1190 à 1210, considérait que les images devaient orner les églises pour l’instruction des laïcs, conformément à la doctrine professée par Grégoire le Grand : «Et nota, quod decorantur ecclesie picturis et sculpturis et inmaginibus et huiusmodi duabus de causis, silicet ut sint ornatus ecclesiarum et ut sint littere laicorum. Littere quidem sunt rememoratione preteritorum, indicative presentium, pronostice futurorum. Preteritorum, ut istoriarum et visionum, presentium, ut virtutum et viciorum, futurorum, ut penarum et premiorum. ${ }^{22}$ C'est ainsi que, pour mieux actualiser l'histoire sainte, l'auteur recommande notamment que les saints soient peints avec leurs attributs respectifs : «Quandoque Io[annes] Bap[tista] interseritur ut eremita, martires cum aculeis, ut Laurentius in craticula et Stephanus in lapidibus, confessores cum suis insignibus, ut episcopi mitrati, abbates capuciati, doctores cum libris in manibus, virgines secundum evangelium cum lampadibus. Quandoque martires cum palmis, confessores cum liliis depinguntur. Palma victoriam, lilium castitatem significat. ${ }^{23}$ D'autant plus intéressant qu'il est de la plume d'un Italien, ce passage témoigne bien de l'effort de codification iconographique entrepris à cette époque par les théologiens et les canonistes. Celui-ci ne fait que se renforcer durant le XIIIe siècle, comme l'attestent les longs développements sur les représentations des saints par Guillaume Durand (1230/31-1296), dans son Rationale divinorum officiorum, rédigé vers 1270-1275. S’inspirant des sommes de Jean Beleth et de Huguccio de Pise, l'évêque de Mende consacre dans son traité un chapitre entier aux images, intitulé « De picturis et cortinis et ornamentis ecclesie $»^{24}$. Ce passage, qui se présente comme un véritable traité d'iconographie religieuse, fit autorité jusqu'à l'époque de la Contre-Réforme, en l'absence d'autres traités postérieurs sur la manière de représenter les scènes religieuses. Mettant en exergue la phrase célèbre de Grégoire le Grand sur les images et insistant sur la dimension affective de

\footnotetext{
${ }^{20}$ Ed. Arthur M. Landgraf, Commentarius Cantabrigiensis. 4. In epistolam ad Hebraeos, Notre Dame, Indiana, 1945, p. 784.

${ }^{21}$ Cap. 164 : «De festo Andree et Tome apostolorum », éd. CCCM 41A, p. 321-322.

22 Bamberg, Staatsbibliothek, Can. 40, f. 265v (passage cité et édité par Arthur M. Landgarf, art. cit.).

${ }^{23}$ Ibid.

${ }^{24}$ Rationale divinorum officiorum I, cap. III, éd. CCCM 140, p. 34-52.
} 
celles-ci ${ }^{25}$, l'auteur reprend les conseils iconographiques de Huguccio de Pise, en les développant de manière plus approfondie. C'est ainsi qu'il précise notamment que saint Paul doit être représenté avec un livre et une épée, car le livre rappelle son activité de docteur ou les circonstances de sa conversion, tandis que l'épée fait référence à son statut de «miles Christi» ${ }^{26}$. De la même façon, il s'intéresse à la forme que revêt l'auréole des saints ou à la signification du dragon de saint Michel $^{27}$. Pour ce théologien, les images des saints doivent être disposées dans les églises et autres lieux saints, de manière à ce que tous puissent méditer sur leurs actions et leur sainteté $^{28}$. Il est donc important que les personnages figurés soient identifiables à l'aide de signes distinctifs, ceux-ci pouvant prendre la forme d'attributs ou de caractéristiques physiques, c'est pourquoi Guillaume Durand insiste également sur l'apparence physique des apôtres Barthélémy et André à l'occasion de leur fête ${ }^{29}$.

Ainsi, l'apparition des attributs individuels dans les représentations des saints au tournant du XIIe siècle s'inscrit au cœur même de l'œuvre pastorale entreprise par l’Église et de la réflexion théologique qui s'est développée à la même époque sur les images ornant les églises et d'autres monuments figurés. Si l'Église n'est jamais réellement parvenue à exercer un contrôle effectif sur l'abondante production d'images aux derniers siècles du Moyen Age, comme en témoignent les nombreux abus iconographiques commis par les artistes de la fin du Moyen Age et dénoncés par les théologiens de la Contre-Réforme ${ }^{30}$, elle n'en a pas moins cherché à codifier dans une certaine mesure les attributs individuels donnés aux saints, pour garantir la conformité des images de ces derniers avec l'histoire sainte elle-même. Cette insistance nouvelle sur les aspects biographiques de la sainteté et sur l'existence terrestre des saints par le biais des attributs, qui trouve son écho dans les thèmes des sermons composés en l'honneur des saints à cette époque, est destinée à proposer aux laïcs des modèles vertueux à suivre. En employant de tels raccourcis visuels, les artistes pouvaient en effet condenser un ensemble de données relatives à la légende d'un saint en une seule image synthétique propre à susciter la piété de ceux qui la contemplent.

Ainsi que nous l'avons vu plus haut, les figures de saints accompagnés de leurs attributs se rencontrent en grand nombre dans les psautiers exécutés au XIIIe siècle dans le Nord de

\footnotetext{
25 « Picture et ornamenta in ecclesia sunt laicorum lectio et scripture, unde Gregorius : Aliud est picturam adorare, aliud per picture ystoriam quid sit adorandum addiscere, nam quod legentibus scriptura hoc ydiotis cernentibus prestat pictura, quia in ipsa ignorantes vident quid sequi debeant, in ipsa legunt qui litteras nesciunt. », Ibid., p. 34-35. « Pictura namque plus videtur movere animam quam scriptura », Ibid., p. 36.

${ }^{26}$ Ibid., p. 40.

${ }^{27}$ Ibid., p. 38 et 41.

${ }^{28}$ Ibid., p. 40.

${ }^{29}$ Ibid., VII, cap. 25, p. 75 et cap. XXXVIII, p. 104.

${ }^{30}$ Sur la liberté des artistes médiévaux, voir notamment la contribution d'André Chastel sur ce sujet, "Le dictum Horatii quidlibet audendi potestas et les artistes ", dans Fables, formes et figures, I, Paris, 2000², p. 363-377.
} 
l'Europe, sous la forme de peintures en pleine page intercalées dans le manuscrit ou d'initiales historiées placées en tête des psaumes 26, 38, 51, 52, 68, 80, 97 et 101. Sans rapport avec le texte qu'elles accompagnent, ces illustrations se présentent en quelque sorte comme des images de dévotion, les attributs jouant un double rôle mnémonique et affectif. Souvent placés côte à côte suivant une disposition quadripartite de la page ou occupant toute la surface de la page, les saints exhibent leur attribut qui a pour fonction de faire méditer les fidèles laïcs sur les hauts faits de la vie des saints comme sur leurs souffrances endurées pour la gloire de Dieu. Citons à titre d'exemple cette peinture en pleine page d'un Psautier-Heures exécuté à Metz vers 1270-1280 (New York, Pierpont Morgan Library, M 88, f. $20^{31}$ ) qui contient, au registre supérieur, sainte Catherine debout, une couronne sur la tête, tenant à la main les instruments de son martyre, l'épée et la roue, la tête couronnée de l'empereur Maximien à ses pieds, et sainte Marguerite émergeant mains jointes des entrailles d'un dragon bicolore tenant encore dans sa gueule un pan de son manteau, et, au registre inférieur, sainte Agnès tenant un livre fermé, un agneau à ses côtés, et sainte Barbe, une tour et une palme dans les mains. De même, une image en pleine page de saint Christophe s'aidant d'un bâton pour traverser les flots avec l'enfant Jésus sur les épaules illustre le Psautier de Yolande de Soissons (New York, Pierpont Morgan Library, M 729, f. $288 \mathrm{v}^{32}$ ), originaire du Nord-Est de la France et daté du dernier quart du XIIIe siècle. Ces deux exemples montrent que les enlumineurs sont parvenus à concilier au sein d'une même image les exigences de la narration et de la contemplation : au lieu de représenter les circonstances d'un épisode de la vie ou du martyre des saints, ils ont utilisé les attributs individuels des saints pour évoquer de manière indirecte le récit et favoriser l'acte de contemplation chez le lecteur grâce à la représentation frontale des personnages munis de leurs « instruments de gloire », sur fond neutre.

Le caractère métonymique des attributs des saints suppose, de la part de celui qui contemple leurs figures, une certaine connaissance des récits hagiographiques, les attributs représentés se contentant de faire brièvement allusion à un épisode de la vie des saints. C'est pourquoi, même après l'apparition de ces signes spécifiques, les artistes ont continué de représenter des scènes illustrant, à l'aide de procédés narratifs, les détails d'un épisode tiré de la légende de saints moins familiers aux fidèles. Aussi est-il fréquent durant le XIIIe siècle de voir coexister au sein d'un même manuscrit des images statiques de saints identifiables par leurs attributs, pour quelques saints universellement connus, et des scènes narratives plus parlantes, pour d'autres. C'est notamment le cas d'une vie de saints du Nord de la France, des XIIIe et XIVe siècles, provenant de Saint-Victor de Paris (Paris, BnF fr. 23117), qui contient une série

\footnotetext{
${ }^{31}$ On trouvera une reproduction de cette miniature dans le CD-Rom de l'Index of Christian Art.

32 On trouvera une reproduction de cette miniature dans le CD-Rom de l'Index of Christian Art.
} 
d'encadrements figurant pour la plupart le martyre des saints et, pour les saints les plus célèbres, les images statiques de ces derniers accompagnés de leurs attributs respectifs : ainsi saint Jacques vêtu en pèlerin et coiffé d'un chapeau, portant un sac timbré d'une coquille et un bourdon (f. 68), saint Jean-Baptiste tenant un clipeus contenant l'agneau crucifère (f. 129) et saint Denis vêtu en évêque, le sommet de sa tête dans les mains (f. 190v). Aux siècles suivants, la diffusion massive des attributs des saints dans l'art a contribué à estomper progressivement cette dualité des procédés narratifs au sein d'un même manuscrit. Ce phénomène est particulièrement sensible dans les livres d'heures du XVe siècle, dans lesquels les scènes de martyre ont généralement cédé la place aux figures de saints accompagnés de leurs attributs.

L’identification des saints dans les images par l'intermédiaire de signes spécifiques dès la seconde moitié du XIIe siècle résulte ainsi des évolutions religieuses survenues à cette époque. Ne pourrait-on cependant mettre également en relation l'apparition des attributs individuels avec un autre phénomène, celui de l'émergence d'autres signes distinctifs à la même époque au sein de la société ? En ce qui concerne les attributs des saints, le vocabulaire occasionnellement employé pour désigner ces nouveaux motifs iconographiques dans les textes médiévaux reflète bien la notion d'identité qui s'y attache, ainsi que l'indique la signification des termes «intersigna», faisant référence à la coquille de l'apôtre saint Jacques dans le Liber sancti Jacobi (v. 1139) $)^{33}$, et d'« insignibus » utilisé par Huguccio de Pise à propos des attributs des saints confesseurs dans la Summa decretorum ${ }^{34}$. Ces deux termes désignent en effet un signe, une marque distinctive, manifestant à la fois l'identité de son possesseur et la gloire de son martyre. Contrairement à toute attente, ils semblent être les seuls utilisés dans ce contexte à l'époque médiévale, les termes «adtributum », «character », «nota» ou «emblema» apparaissant dans des contextes très différents. La volonté de caractériser les saints à l'aide de signes est également manifeste dans les esquisses et annotations marginales d'un manuscrit de la légende dorée, réalisé à Paris aux environs de 1280-1290 (San Marino, Huntigton Library, HM 3027) ${ }^{35}$ : en regard de la miniature

\footnotetext{
${ }^{33} \mathrm{~J}$. Vielliard, Le guide du pèlerin de saint Jacques de Compostelle, 5e éd., Mâcon, 1978, p. 96 (lib. V, cap. IX, \ 6) : « Post fontem habetur paradisus, ut diximus, pavimento lapideo factus, in quo crusille piscium id est intersigna beati Jacobi venduntur peregrinis...».

${ }^{34}$ Huguccio de Pise, Op. cit., Bamberg, Staatsbibliothek, Can. 40, f. 265v.

35 Sur ce manuscrit, voir l'article de Jonathan J.G. Alexander, "Preliminary marginal drawings in medieval manuscripts ", paru dans Artistes, artisans et production artistique au Moyen Age, Paris, 1986, III, p. $307-319$ (cf. en particulier les pages 308-309 et l'illustration $\mathrm{n}^{\circ} 2$ ). Nous sommes également très reconnaissante à Alison Stones de nous avoir gracieusement fait part de ses informations sur ce manuscrit.
} 
du f. $118 v$ représentant deux chasseurs à cheval, l'un sonnant du cor, l'autre bandant son arc en direction d'un cerf venu trouver refuge sur les genoux de saint Gilles assis, habillé en moine, une note en bas de page donne les directives suivantes à l'enlumineur: "sanctus egidius cum cerva ». La précision « cum cerva », qui se rapporte au contenu de l’image, est destinée à rappeler à l'enlumineur qu'il devait également représenter l'attribut spécifique de saint Gilles, une biche (transformée ici en un cerf).

Ces quelques exemples littéraires et iconographiques illustrent bien la volonté qui s'est manifestée dès le milieu du XIIe siècle d'identifier présisément les saints dans les images, les attributs jouant le rôle d'un «surnom ». Ce phénomène ne pourrait-il ainsi être mis en relation avec l'apparition, au cours du XIIe siècle, des armoiries au sein de la noblesse et d'un système de dénomination à deux éléments, pour mieux différencier entre eux les membres du corps social ?

Ainsi que l'a souligné Michel Pastoureau, les armoiries constituent une nouvelle formule emblématique apparue en Europe occidentale durant la première moitié du XIIe siècle, et qui s'est d'abord diffusée principalement dans les régions situées entre la Loire et la Meuse ${ }^{36}$. Distinguant trois phases dans le processus d'élaboration des armoiries, Michel Pastoureau a montré que celles-ci sont apparues entre 1120-1130 et 1160-1170, après une phase de gestation allant du début du XIe siècle aux années 1120-1130, et se sont ensuite largement diffusées entre 1170 et 1230. Analysé par Brigitte Bedos-Rezak dans le cadre d'une réflexion plus vaste sur les rapports unissant l'individu et la société, l'exemple des sceaux est à cet égard éclairant ${ }^{37}$ : ces derniers, qui constituent un support plastique privilégié pour la diffusion des armoiries, commencèrent à être ornés d'armoiries en Ile-de-France et en Picardie à partir des années 1130. L'apparition de ce système emblématique s'inscrit dans le bouleversement des structures sociales de l'époque : l'établissement de l'ordre seigneurial, caractérisé par l' «encellulement» des classes et des catégories sociales suivant le concept défini par Robert Fossier, rendit nécessaire l'identification de chacun à l'aide de nouveaux signes d'identité, les anciens, qui s'appuyaient sur un ordre social disparu, étant devenus obsolètes. Dans ce contexte, les individus appartenant à l'aristocratie se dotèrent d'armoiries qui indiquaient non seulement leur position sociale, mais aussi leur lignage.

La naissance d'un système de dénomination à deux éléments relève également de ce fait de civilisation. Mis en lumière par Karl F. Werner puis par Monique Bourin à partir d'une série

\footnotetext{
${ }^{36}$ Michel Pastoureau, "La naissance des armoiries », dans Le XIIe siècle. Mutations et renouveau en France dans la première moitié du XIIe siècle, Cahiers du Léopard d'or n³, Paris, 1994, p. 103-122.

${ }^{37}$ Brigitte Bedos-Rezak, «L'apparition des armoiries sur le sceaux en Ile-de-France et en Picardie (v. 1130-1230)», dans Les origines des armoiries. Actes du 2e colloque de l'Académie internationale d'héraldique, Bressanone / Brixen, 5-9 septembre 1981, éd. H. Pinoteau, M. Pastoureau et M. Popoff, Paris, 1983, p. 23-41. B. Bedos-Rezak est par ailleurs l'auteur d'une étude stimulante sur la notion d'identité au Moyen Age : "Medieval identity : a sign and a concept», dans American historical review, vol. 105, n5 (déc. 2000), p. 1489-1533.
} 
d'enquêtes régionales reposant sur le dépouillement de cartulaires du XIe siècle au XIIIe siècle ${ }^{38}$, ce phénomène est devenu manifeste au cours du second quart du XIIe siècle et, dès les années 1160-1170, la majorité des individus furent désignés par un nom double, le nom propre et le surnom, ce dernier pouvant faire référence aussi bien à l'ascendance paternelle et à l'ancêtre éponyme qu'au lieu de résidence de l'individu. Ainsi, grâce aux armoiries et au nom patronymique, les individus et les groupes sociaux eurent la possibibilité d'affirmer leur identité au sein de cette nouvelle société.

Il en va de même pour les attributs iconographiques des saints, qui remplissent la même fonction que les armoiries ou les patronymes. A cet égard, on notera les points de convergence entre la chronologie de l'apparition des armoiries, des noms patronymiques et des attributs individuels en Occident durant le XIIe siècle, de même qu'entre l'aire de la diffusion des armoiries et celle des attributs individuels, plus répandus au Nord qu'au Sud de l'Europe. En outre, à l'instar des armoiries, les attributs des saints utilisent un langage restreint de formes et de couleurs possédant une signification précise, telles les deux clefs de saint Pierre que les artistes ont parfois distinguées au moyen de couleurs différentes pour mieux souligner la différence entre le pouvoir spirituel et le pouvoir temporel du saint ${ }^{39}$, ou le dragon de sainte Marguerite, dont l'aspect bariolé et tacheté revêt une connotation nettement péjorative. La diffusion des attributs iconographiques des saints, à la même époque que celle des armoiries et des patronymes, reflète ainsi une certaine évolution dans la notion de sainteté : perçu de plus en plus comme un personnage concret, le saint reçoit ainsi comme les autres membres du corps social des signes de reconnaissance empruntés à sa vie terrestre, à ses vertus ou à son culte, qui l'identifient et l'ancrent plus profondément dans la société religieuse et profane.

Par conséquent, l'apparition des attributs individuels semble bien participer non seulement d'un phénomène religieux, mais aussi d'un phénomène social plus large, celui de l'affirmation de l'identité des individus au sein d'une société «suremblématisée ». Désormais, les

\footnotetext{
${ }^{38}$ Karl F. Werner, «Liens de parenté et noms de personne », dans Famille et parenté dans l'Occident médiéval. Actes du colloque de Paris (6-8 juin 1974), E.P.H.E., VIe section, Rome, 1977, p. 13-18 et 25-34 (cf. en particulier p. 26-27) ; Monique Bourin, «Bilan de l'enquête : de la Picardie au Portugal, l'apparition du système anthroponymique à deux éléments et ses nuances régionales », dans Genèse médiévale de l'anthroponymie moderne, I, Tours, 1987, p. 233-246.

39 On signalera notamment une représentation de saint Pierre tenant deux clefs, l'une verte l'autre blanche, de taille et de forme différentes, dans l'initiale I ornant le f. 10 d'un Liber pontificalis, exécuté à Saint-Evroult d'Ouche entre 1113 et 1133 (Rouen, BM, ms. 31). De même, dans une Bible anglaise, vers 1245-1255, saint Pierre tient une clef jaune et une clef rouge dans les mains (Angers, BM, ms. 9, f. 261).
} 
attributs constituent des signes distinctifs, des éléments de repère permettant d'identifier avec certitude les saints dans les images. Cette emblématisation des attributs des saints atteignit son point culminant à la fin du Moyen Age, avec l'adoption par des grands personnages de l'attribut de leur saint patron comme emblème - que l'on pense par exemple à l'omniprésence de la croix de saint André dans l'iconographie de la maison de Bourgogne au XVe siècle -, comme avec la fabrication dans le Nord de l'Europe de calendriers médiévaux sur bois, sur lesquels la fête de chaque saint est signalée uniquement par la représentation de son attribut conventionnel ${ }^{40}$. Mais ceci est un autre chapitre...

Charlotte Denoël

${ }^{40}$ On trouvera les références de ces calendriers dans l'article d'Alois Riegl, «Die holzkalender des Mittelalters und der Renaissance », dans Mitteilungen des Instituts für österreiscbiche Geschicbtsforschung, IX (1888), 82-103. 